\title{
An Unusual Location of a Primary Central Nervous System Lymphoma: The Temporal Horn
}

\author{
Hyok-Rae Cho
}

\begin{abstract}
Primary central nervous system lymphomas (PCNSLs) are commonly seen at the cerebral hemisphere, especially the corpus callosum, basal ganglia and thalamus. It is extremely rare for a PCNSL to occur in the temporal horn among pathology of intraventricular tumors. This patient was a 59-year-old man and referred our hospital due to confusion and gait disturbance. On the enhanced MRI, homogeneous-enhanced solitary mass was found within the temporal horn of the left lateral ventricle. That lesion was accompanied with increasing hypervascular tumor blush on the preoperative cerebral angiography. The author chose a craniotomy for removal of mass and tissue diagnosis and reported a patient with an extraordinary diagnosis of PCNSL at the temporal horn.
\end{abstract}

Keywords: Lymphoma; Temporal horn; Central nervous system; Location

\section{Introduction}

Primary central nervous system lymphoma (PCNSL) is an uncommon but aggressive intracranial malignant in adults. Usually, lesion is located at cerebral hemisphere although PCNSL can affect any part of the neuraxis. It accounts for $2.2 \%$ of all central nervous system (CNS) tumors [1].

Tumor neurosurgeons have typically preferred to diagnose this tumor by biopsy, rather than resection according to preoperative workup. Careful consideration of the brain MRI and angiography was integral to diagnosing our patient. At the current case, author intends to describe that PCNSL was unexpectedly found at the temporal horn.

\section{Case Report}

A 59-year-old man presented with a several days history of

Manuscript accepted for publication April 16, 2015

Department of Neurosurgery, Kosin University Gospel Hospital, 262 Gamcheon-ro, Seo-gu, 602-702 Pusan, Korea. Email: drchr@hanmail.net

doi: http://dx.doi.org/10.14740/jmc2155w confusion and gait disturbance. His past history included cerebellar hemangioblastoma operation at the outside hospital before 10 years ago. On examination, the patient was in confusion, with no cranial nerve deficit. He displayed unsteady gait. His motor strength was normal.

MRI revealed a $3 \mathrm{~cm}$ temporal horn mass of oval shape on T1-weighted sequences, with a homogenous contrast enhancement after gadolinium administration. T2-weighted imaging highlighted a surrounding edema around the tumor (Fig. 1). Preoperatively, the author ascertained whether the tumor was hypervascular at the cerebral angiography (Fig. 2). This case was not candidate for stereotactic biopsy due to prominent vascular blush of tumor at preoperative angiography and tumor location. He was commenced with a preoperative dexamethasone and loaded with the prophylactic valprate anticonvulsant therapy.

At craniotomy, the patient was placed in the supine under general anesthesia. The head was flexed and then turned $30^{\circ}$ to the contralateral side and placed in a three-pint fixation using Mayfield head frame. The skin incision was begun $1 \mathrm{~cm}$ anterior to the tragus at the level of the zygoma extending initially vertically and posteriorly and then gently curving over the superior temporal line to end at the midline behind the hairline (Fig. 3). The dura was also opened into the semicircular fashion. The sylvian fissure was widely split using a combination of sharp and blunt dissection and the basal cisterns were opened for exposing the middle cerebral artery fully. After the inferior circular sulcus of the insula and the limen insula were satisfactorily exposed, a $30 \mathrm{~mm}$ incision was made along the inferior circular sulcus of the insula $1 \mathrm{~cm}$ posteriorly to the limen insula. Dissecting bottom of the inferior circular sulcus of the insula disclosed the underlying tumor in the left temporal horn (Fig. 4). The lesion appeared gray-whitish, soft in consistency, and prone to bleed. The subtotal removal of the tumor was only undergone with use of the ultrasonic surgical aspirator. That was because a frozen biopsy identified lymphoma during the procedure. Postoperative neurological status of this patient was no change and he was referred to oncology department for further evaluation and adjuvant chemotherapy. Histopathology confirmed a malignant lymphoma, non-Hodgkin, diffuse large B cell (Fig. 5). And adjuvant chemotherapy improved clinical outcome.

Postoperative MR study was done 3 months after the surgery. The postoperative images showed complete disappearance of the temporal horn tumor (Fig. 6). The Karnofsky performance scale of this patient was 90 . 

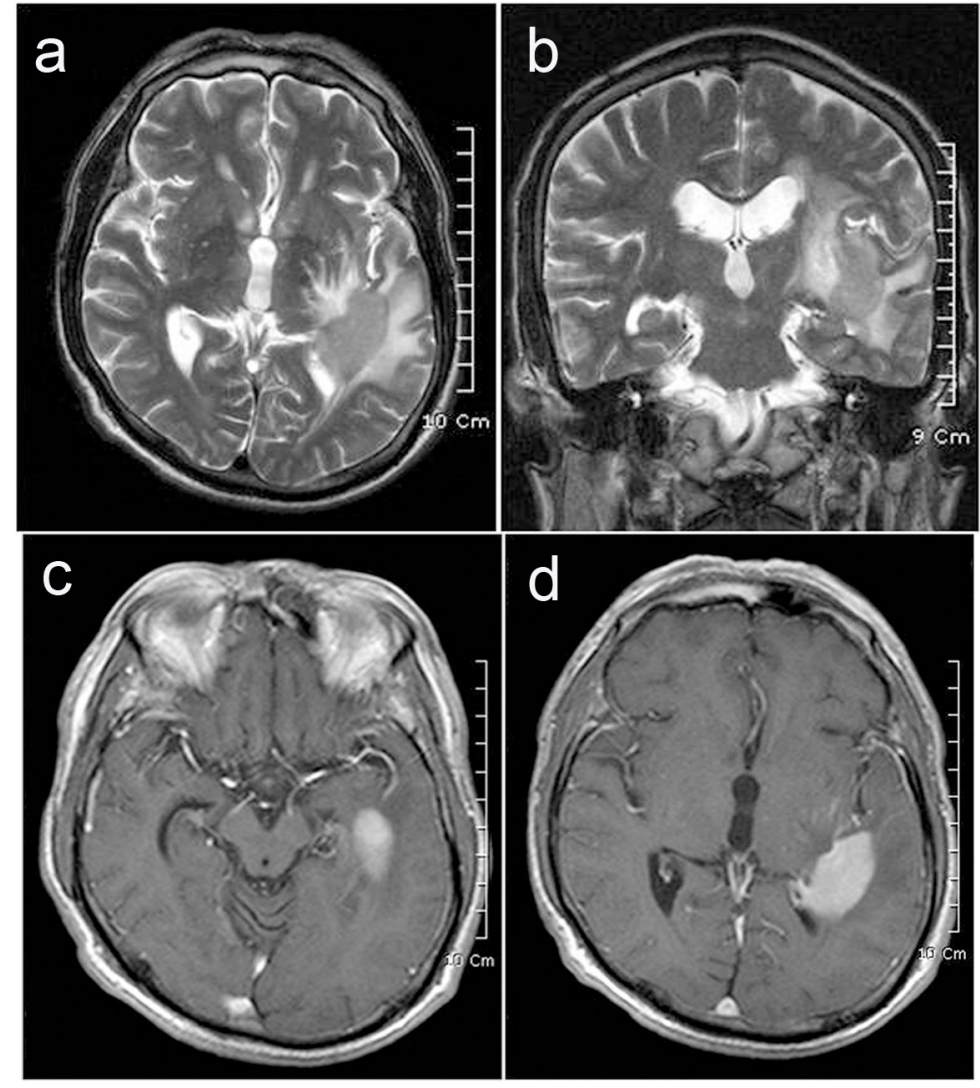

Figure 1. Preoperative non-enhanced axial (a), coronal (b) and enhanced axial (c, d) MRI scans demonstrate a large tumor of ovoid shape inside the left temporal horn with homogenous enhancement and peritumoral edema.

\section{Discussion}

PCNSL is rare type of an extra-nodal non-Hodgkin's lymphoma (NHL), which is confined to the CNS. It arises within

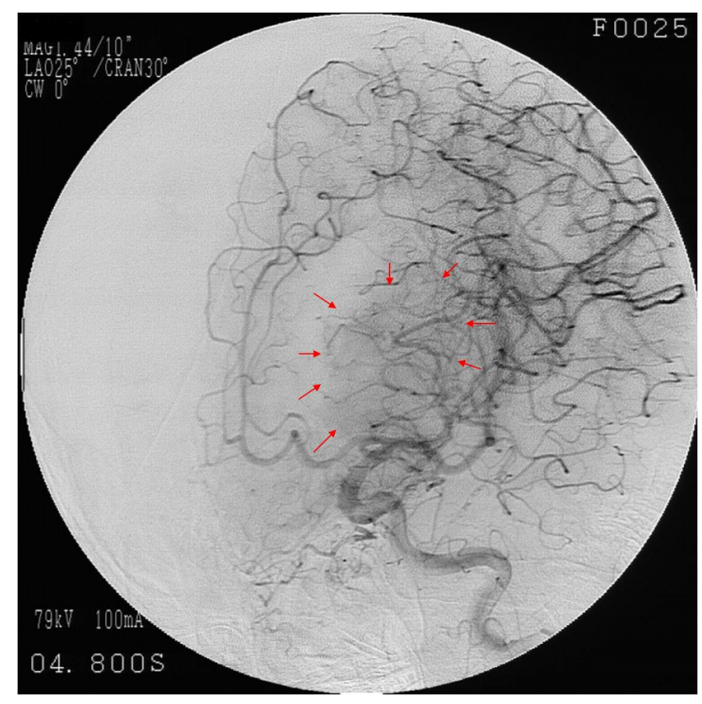

Figure 2. Preoperative angiogram reveals significant tumor vascularity (red arrow). the brain, eyes, leptomeninges, or spinal cord. It accounts for approximately $2.2 \%$ of all the primary CNS tumors and less than $1-4 \%$ of all NHLs with $90-95 \%$ classified histologically as diffuse large B-cell lymphoma. The majority of PCNSLs are sporadic and incidence increases with age. In recent years, its

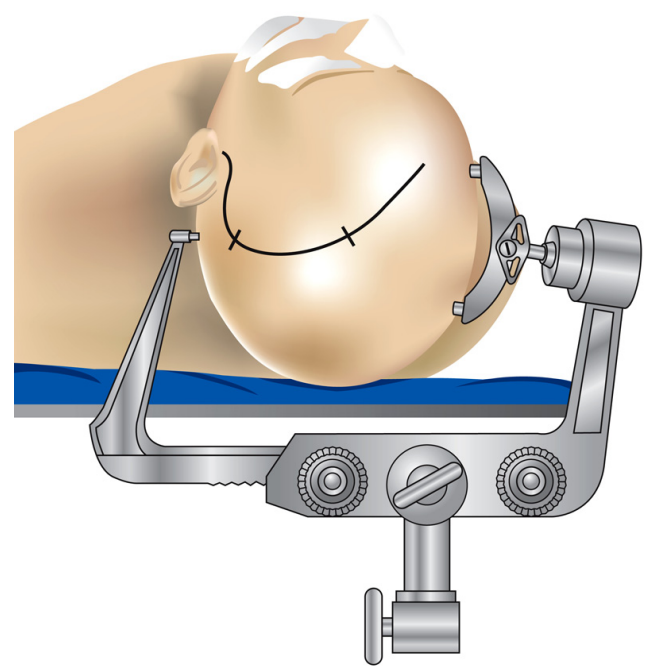

Figure 3. Head position and the skin incision for the pterional-transsylvian approach. 

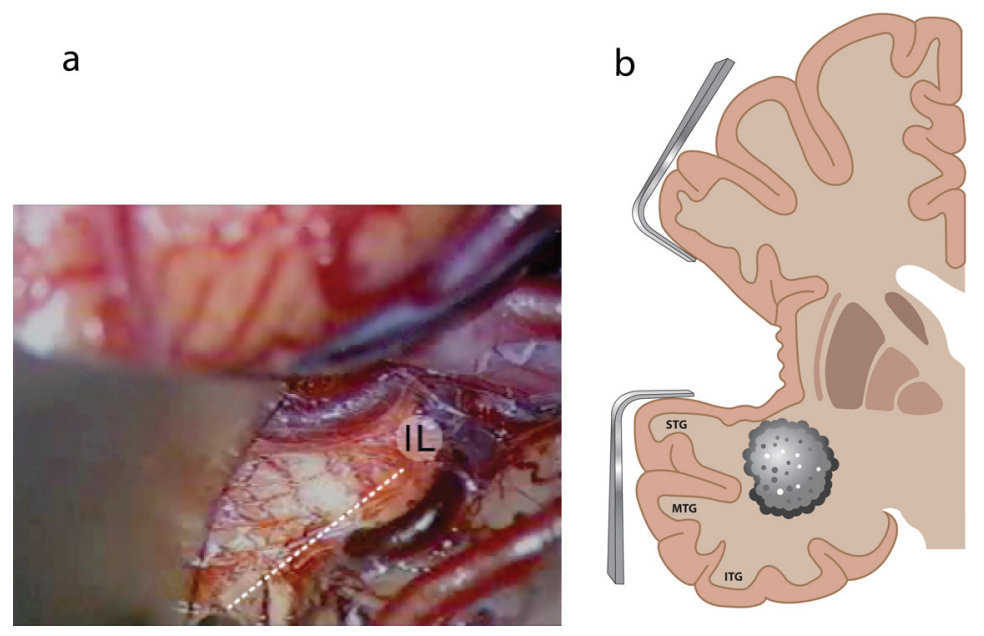

Figure 4. (a) The sylvian fissure is split to identify the inferior insular sulcus. The inferior insular sulcus was mapped with dotted white lines. (b) The temporal horn is reached by dissecting the bottom of the inferior circular sulcus of the insula. IL: insula lumen; STG: superior temporal gyrus; MTG: middle temporal gyrus; ITG: inferior temporal gyrus.

frequency has trebled in immunocompetent individuals. A remaining portion is associated with immunosuppressed states, including HIV infection or iatrogenic immunosuppression following organ transplantation [1].

In a review article by Gerstner and Batchelor, an MRI series of 100 patients revealed that lesions were solitary in $65 \%$ of patients and multifocal in 35\% of patients. And lesions were located in the hemispheres (38\%), thalamus/basal ganglia (16\%), corpus callosum (14\%), periventricular lesion (12\%), and cerebellum (9\%) [2]. Among these, the incidence of intraventricular PCNSL was still unknown. And, intraventricular lymphoma was mainly found in the body of lateral ventricle [3]. In a clinical MRI study of Kuker et al, intraventricular lymphoma, i.e. mass lesions originating from the ventricular borders protruding into the internal CSF spaces, were found in nine of 100 patients. The finding was that exophytic tumor was considered to be extending into the ventricular lumen. Therefore, PCNSL with intraventricular lesion may be a distinct subform of cerebral lymphoma [4].

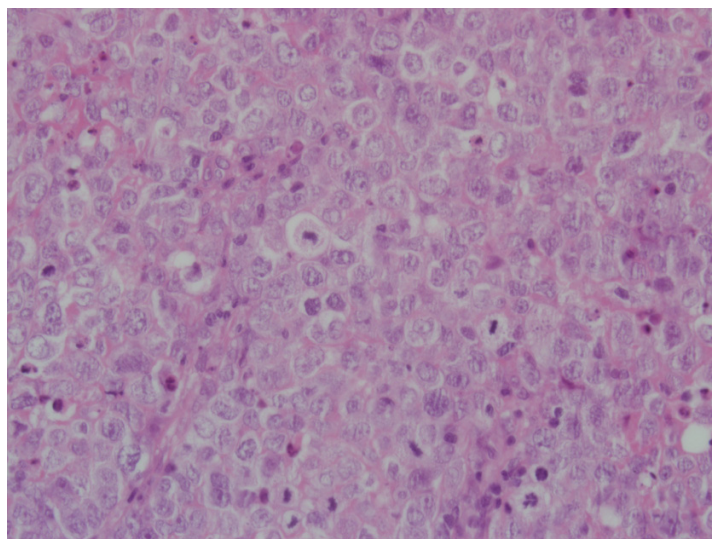

Figure 5. Photomicrograph showing malignant lymphoid infiltrate with perivascular lesion (hematoxylin and eosin, original magnification, $\times$ 400).
MRI is the principal modality for the detection of PCNSL and recent publications have focused on the diagnostic role of advanced MRI techniques. Yap et al illustrated MR features of PCNSL in the immunocompetent patient over a 5-year period at their institution. The typical features of PCNSL were intra-axial homogenous single or multiple contrast enhancing lesions, with marked surrounding edema and restricted diffusion, usually contacting a cerebrospinal fluid (CSF) surface. The frontal lobe was most frequently involved [5]. In this case, MR finding of temporal horn tumor was corresponded to intra-axial single lesion of ovoid shape with homogenous enhancement and surrounding edema. On the other hand, tumor location was unusual site. Preoperative MRI was unable to distinguish intraventricular solitary PCNSL from other primary intraventricular neoplasms and did not add to the specificity of the differential diagnosis.

Consequently, tissue diagnosis was required for current case. Author preferred open surgical resection and biopsy to stereotactic needle biopsy due to hemorrhage risk. That lesion was accompanied with increasing hypervascular blush on preoperative cerebral angiography.

The various types of tumor also occurred in the lateral ventricle $[3,6]$. Ependymoma, subependymoma, central neurocytoma, low-grade glioma, high-grade glioma, choroid plexus papilloma, meningioma and epidermoid cyst were considered to be diagnostic candidates before surgery.

In a retrospective study by D'Angelo et al, the surgical approach to the lateral ventricle tumors was individualized and based on the basis of tumor origin and development: primary or secondary ventricular and associated transependymal development [7]. Coppens et al described three surgical routes to the mesial temporal lobe structures and the temporal horn according to the direction and surface. They included a transsylvian approach, a lateral transsulcal or transcortical approach through the superior or inferior temporal sulcus and their adjacent gyri, and a subtemporal approach through the parahippocampal or fusiform gyri [8]. 


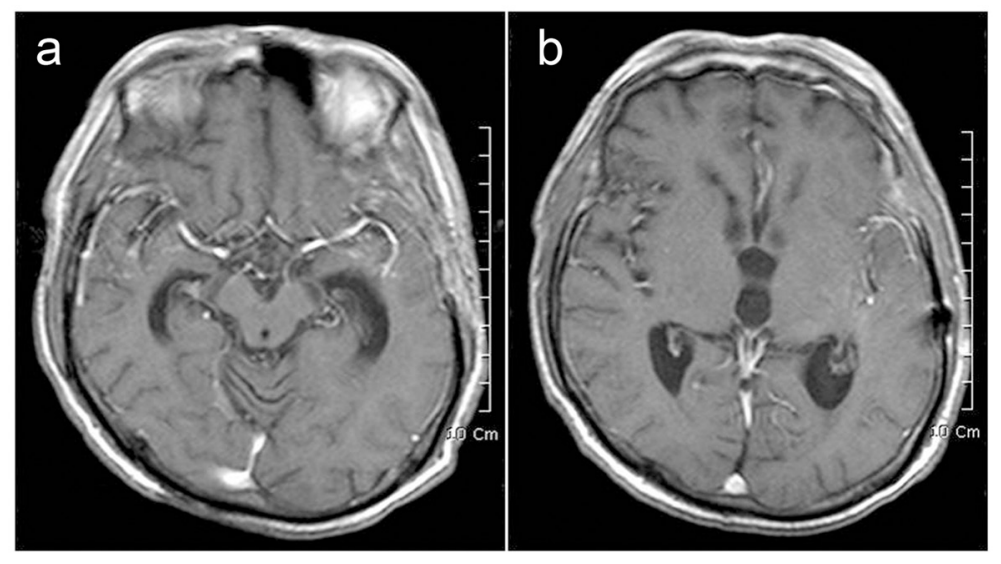

Figure 6. Postoperative enhanced MRI in axial $(a, b)$ views 3 months after operation and chemotherapy shows the absence of tumor.

Morbidity associated with surgery routes to the temporal horn consists mainly of visual field deficits and aphasia. The injury of the optic radiation and the uncinate fasciculus are closely related with visual field deficits and aphasia. Although the subtemporal approach is very effective at visual field preservation because the entrance to the temporal horn is through inferior wall, a significant degree of retraction has to be performed to give adequate access to the occipitotemporal sulcus and can lead to contusions or injury to the vein of Labbe.

Consequently, author chose the transsylvian approach to avoid postoperative complications.

For the transsylvian approach, the inferior circular sulcus of the insula and the limen insula are important landmarks to enter the temporal horn. Intraoperative image shows that inferior insular sulcus was mapped with white dotted lines (Fig. 4a). Under normal conditions, the temporal horn can be reached by dissecting the bottom of the inferior circular sulcus of the insula, following its direction, $1 \mathrm{~cm}$ posteriorly to the limen insula (Fig. 4b). The more posteriorly is the dissecting site in the inferior circular sulcus, the easier could be the entry into the temporal horn.

An effective standard care for PCNSL has been difficult to establish and is the subject of ongoing debate. The individual modalities of treatment include corticosteroid, radiation, chemotherapy, intrathecal chemotherapy, high-dose chemotherapy with stem cell rescue, salvage therapy.

\section{Conclusion}

Incidence of PCNSL is rare in the ventricular system, especially the temporal horn. In a patient whose MR scan shows findings suggestive of PCNLS, a needle biopsy is a rational step in the workup of PCNSL. But, recognizing PCNSL by only imaging criteria is not ease in the temporal horn. Tumor surgeon may easily overlooked ventricle tumor. From our experience, craniotomy is ascertained as the best surgical procedure for the diagnosis of PCNSL among temporal horn tumors.

\section{References}

1. Phillips EH, Fox CP, Cwynarski K. Primary CNS lymphoma. Curr Hematol Malig Rep. 2014;9(3):243-253.

2. Gerstner ER, Batchelor TT. Primary central nervous system lymphoma. Arch Neurol. 2010;67(3):291-297.

3. Jelinek J, Smirniotopoulos JG, Parisi JE, Kanzer M. Lateral ventricular neoplasms of the brain: differential diagnosis based on clinical, CT, and MR findings. AJR Am J Roentgenol. 1990;155(2):365-372.

4. Kuker W, Nagele T, Korfel A, Heckl S, Thiel E, Bamberg $\mathrm{M}$, Weller M, et al. Primary central nervous system lymphomas (PCNSL): MRI features at presentation in 100 patients. J Neurooncol. 2005;72(2):169-177.

5. Yap KK, Sutherland T, Liew E, Tartaglia CJ, Pang M, Trost N. Magnetic resonance features of primary central nervous system lymphoma in the immunocompetent patient: a pictorial essay. J Med Imaging Radiat Oncol. 2012;56(2):179-186.

6. Fenchel M, Beschorner R, Naegele T, Korn A, Ernemann U, Horger M. Primarily solid intraventricular brain tumor. Eur J Radiol. 2012;81(4):688-696.

7. D'Angelo VA, Galarza M, Catapano D, Monte V, Bisceglia M, Carosi I. Lateral ventricle tumors: surgical strategies according to tumor origin and development--a series of 72 cases. Neurosurgery. 2005;56(1 Suppl):36-45; discussion 36-45.

8. Coppens JR, Mahaney KB, Abdulrauf SI. An anteromedial approach to the temporal horn to avoid injury to the optic radiation fibers and uncinate fasciculus: anatomical and technical note. Neurosurg Focus. 2005;18(6B):E3. 